\title{
Is hyperuricemia an independent risk factor for new-onset chronic kidney disease?: a systematic review and meta-analysis based on observational cohort studies
}

\author{
Ling $\mathrm{Li}^{1}$, Chen Yang ${ }^{2}$, Yuliang Zhao ${ }^{1}$, Xiaoxi Zeng ${ }^{1}$, Fang Liu ${ }^{1}$ and Ping Fu ${ }^{1 *}$
}

\begin{abstract}
Background: Hyperuricemia has been reported to be associated with chronic kidney disease (CKD). However whether an elevated serum uric acid level is an independent risk factor for new-onset CKD remained controversial.

Methods: A systematic review and meta-analysis using a literature search of online databases including PubMed, Embase, Ovid and ISI Web/Web of Science was conducted. Summary adjusted odds ratios with corresponding 95\% confidence intervals $(95 \% \mathrm{Cl})$ were calculated to evaluate the risk estimates of hyperuricemia for new-onset CKD.

Results: Thirteen studies containing 190,718 participants were included. A significant positive association was found between elevated serum uric acid levels and new-onset CKD at follow-up (summary OR, 1.15; 95\% Cl, 1.05-1.25). Hyperuricemia was found be an independent predictor for the development of newly diagnosed CKD in non-CKD patients (summary $\mathrm{OR}, 2.35 ; 95 \% \mathrm{Cl}, 1.59-3.46)$. This association increased with increasing length of follow-up. No significant differences were found for risk estimates of the associations between elevated serum uric acid levels and developing CKD between males and females.
\end{abstract}

Conclusions: With long-term follow-up of non-CKD individuals, elevated serum uric acid levels showed an increased risk for the development of chronic renal dysfunction.

Keywords: Chronic kidney disease, Hyperuricemia, Uric acid

\section{Background}

The prevalence of chronic kidney disease (CKD) is increasing in various populations worldwide, and risk factors associated with CKD have gained much attention in recent years [1]. Hypertension, lifestyle and metabolic syndrome among other factors have been associated with the incidence of CKD in previous studies [2-4]. Since the 1990s, nephrologists have reported that hyperuricemia is associated with CKD. However, other risk factors such as hypertension, metabolic syndrome and gout have also been proven to be associated with hyperuricemia [5]. Therefore, whether an elevated uric acid level is an independent risk factor for CKD remains to

\footnotetext{
*Correspondence: fupinghx@163.com

'Division of Nephrology, West China Hospital of Sichuan University, Chengdu 610041, Sichuan, China

Full list of author information is available at the end of the article
}

be elucidated. Increasing evidence suggests that hyperuricemia may be a pathogenic factor for the development of CKD rather than just a marker of decreased renal uric acid excretion. Several potential mechanisms have been suggested to explain the causal relationship between hyperuricemia and CKD, including vascular smooth cell proliferation [6], endothelial dysfunction [7], increased synthesis of interleukin-6, impaired endothelial nitric oxide production [8] and insulin resistance [9]. In the past 3 years, etiologic studies have explored relationships between hyperuricemia and renal disease, and most studies revealed a positive association between elevated uric acid levels and CKD or new-onset CKD [10]. Some nephrologists recommend management of uric acid in CKD patients or groups at high risk of CKD [11].

The current meta-analysis was performed to quantitatively assess relationships between hyperuricemia and 
CKD, and also to evaluate whether associations were influenced by hypertension, hyperuricemia or other confounding factors, and whether they varied by study design, sex and geographical area.

\section{Methods}

Study selection

A literature search was performed using electronic databases Medline Ovid/Medline (1948 to present), PubMed/ Medline, Embase, and ISI Web/Web of Science for published studies from January 1970 to September 2013. Key words used were: "hyperuricemia", "uric acid elevated", "hyperuricaemia", "uricacidemia", and "uricacidaemia"; and "CKD" or "chronic kidney disease" or "chronic renal dysfunction" or "chronic renal insufficiency". The "related items" function was used to broaden the search. The electronic search was up to September 2013 with no limitations regarding the type of publication. Studies were screened by two reviewers (L.L. and C.Y.) independently with the following inclusion criteria: (1) report of the association between serum uric acid (SUA) and new-onset CKD; (2) studies of observational cohort design; and (3) report of the hazard ratio (HR) or odds ratio (OR) in cohort studies with $95 \%$ confidence intervals (95\% CI) or sufficient information to calculate these figures. Exclusion criteria were: (1) studies published in languages other than English; and (2) studies reporting on patients with end-stage renal disease or acute renal impairment or patients requiring dialysis. The definition of CKD was: eGFR $<60 \mathrm{~mL} / \mathrm{min}$ per $1.73 \mathrm{~m}^{2}$ for more than 3 months or the presence of albuminuria (kidney damage) for more than 3 months, according to the National Kidney Foundation Kidney Disease Outcomes and Quality Initiative (KDOQI) CKD guidelines [12]. Studies that used other definitions for CKD, such as serum creatinine levels, were excluded. If more than one study reported duplicated material, the most recent study was included in the systematic review. Reference lists of all included studies were reviewed for any additional relevant studies. The whole process of this systematic review was conducted in strict accordance with the criteria of the moose checklist to ensure the quality of this study (Additional file 1).

\section{Outcome measure}

KDOQI CKD guidelines were used for the definition of CKD severity: Stage 1, GFR $>90 \mathrm{~mL} / \mathrm{min}$ per $1.73 \mathrm{~m}^{2}$ with microalbuminuria or macroalbuminuria; Stage 2, GFR 60$89 \mathrm{~mL} / \mathrm{min}$ per $1.73 \mathrm{~m}^{2}$ with microalbuminuria or macroalbuminuria; Stage 3, GFR 30-59 mL/min per $1.73 \mathrm{~m}^{2}$; and Stage 4 GFR 15-29 mL/min per $1.73 \mathrm{~m}^{2}$ [12]. Two reviewers (L.L. and C.Y.) retrieved the following data from the included studies independently: author, year published, country where study was performed, material source, cut-off level for hyperuricemia, sample size (number of patients and controls), SUA levels in CKD and non-CKD groups (mean $\pm \mathrm{SD}$ ), variables adjusted for calculation in the analysis, and adjusted OR or HR estimates with corresponding 95\% CI. If the study classified participants according to quartiles of uric acid level, the highest quartile was taken to indicate hyperuricemia. Data reporting on the OR or HR of hyperuricemia and new-onset CKD or CKD risk were retrieved and combined.

\section{Quality assessment}

The Newcastle-Ottawa Scale (NOS) was used for metaanalysis of observational studies in epidemiology for assessment of quality [13]. Risk of bias was assessed among the cohort studies on three aspects: (1) selection of participants (containing four domains); (2) comparability (one domain); and (3) outcome measure (containing three domains). Each domain was rated as "Yes", "No", or "Unclear". Each quality domain was categorized as low risk for bias (Yes) when adequate data were reported to assess the study quality or the study meeting the criteria, and high risk (No) when the study reported adequate data but did not meet the criteria for that quality domain. Studies that did not report data to assess quality were categorized as "Unclear" and thus potentially at high risk of bias. "Yes" was scored 1 and "No" or "Unclear" were scored " 0 ". A quality bar was plotted for each domain to examine the limitations of the studies. Studies of high quality were defined as score $>5$ points.

\section{Statistical analysis}

Statistical analysis was performed in accordance with The Cochrane Library handbook and the Quality of Reporting of Meta-Analyses guidelines [14,15]. Different measures for risk estimates from multiply analyses, such as HR (Cox regression) or OR (logistic regression), were extracted from studies; only the most adjusted risk estimates that examined associations between SUA level or hyperuricemia and new-onset CKD were extracted and pooled. We used the random effects model for pooled analysis of adjusted odds ratios (AOR) because of anticipated statistical heterogeneity, but the fixed effects model was also used to ensure robustness of the model chosen and susceptibility to outliers. Heterogeneity among studies was assessed using the $\chi^{2}$ test. $P$-values $<0.05$ were considered statistically significant.

Meta-regression analysis was conducted to explore heterogeneity among studies. Possible sources of heterogeneity included participant-specific characteristics such as age, sex, location, and source of participants; and study-specific characteristics such as data type for calculating HR or OR (continuous variables or dichotomous variables), sample size, study quality, and definitions used for hyperuricemia. Funnel plots and Egger regression tests were used to investigate publication bias. We 
used RevMan version 5.1 (The Cochrane Collaboration, The Nordic Cochrane Centre, Copenhagen, Denmark) and Stata version 11.0 for Windows (Stata Corp., College Station, TX, USA) for data analysis and graph formation.

\section{Results}

Search results

We identified 1662 relevant citations in the combined search of online electronic databases (partial overlap) and 21 additional citations from the reference lists of relevant articles. After removing duplicated records, 790 studies were screened by examining titles and abstracts, and 129 relevant studies after primary selection were reviewed by reading the complete text for eligibility. There were 41 studies that reported on associations between SUA and renal disease, and these were assessed in detail. Articles with a cross-sectional or case-control style, a different definition of CKD or insufficient data were excluded. Finally, 13 studies that met inclusion criteria were included in our meta-analysis (Figure 1).

\section{Study characteristics}

The characteristics of the 13 studies are detailed in Table 1. A total of 190,718 participants were included, with sample sizes ranging from 324 to 94,422 . Four studies included the United States or populations of Western countries, and nine studies were conducted in Asian populations.
Nine studies reported the relationship between increasing SUA level and the development of new-onset CKD during follow-up of non-CKD participants (AOR calculated on continuous variables per $1 \mathrm{mg} / \mathrm{dL}$ ), and seven studies presented risk estimates for associations between hyperuricemia and the incidence of CKD (AOR calculated on dichotomous variables). The NOS quality assessment of the included cohort studies is listed in the Additional file 2. Eight cohort studies showed a comparatively high quality $(\mathrm{NOS}>5)$.

The main limitation observed in the majority of studies was unclear reporting of dropout rates, as many studies did not mention uncompleted follow-ups.

Four studies provided the AOR for uric acid level as a risk factor for the development of CKD in males and females separately. Three studies grouped participants in quartiles according to uric acid level (the OR calculated for the highest quartile compared with the lowest quartile as a reference was retrieved for pooled analysis). Eleven studies defined CKD as the development of eGFR $<60 \mathrm{~mL} / \mathrm{min}$ per $1.73 \mathrm{~m}^{2}$, while the remaining two used albuminuria for the definition.

With regard to choice of participants, 11 studies used population-based participants who underwent health examinations, while the source in the other two studies was hospital-based participants with diabetes mellitus [18,24].

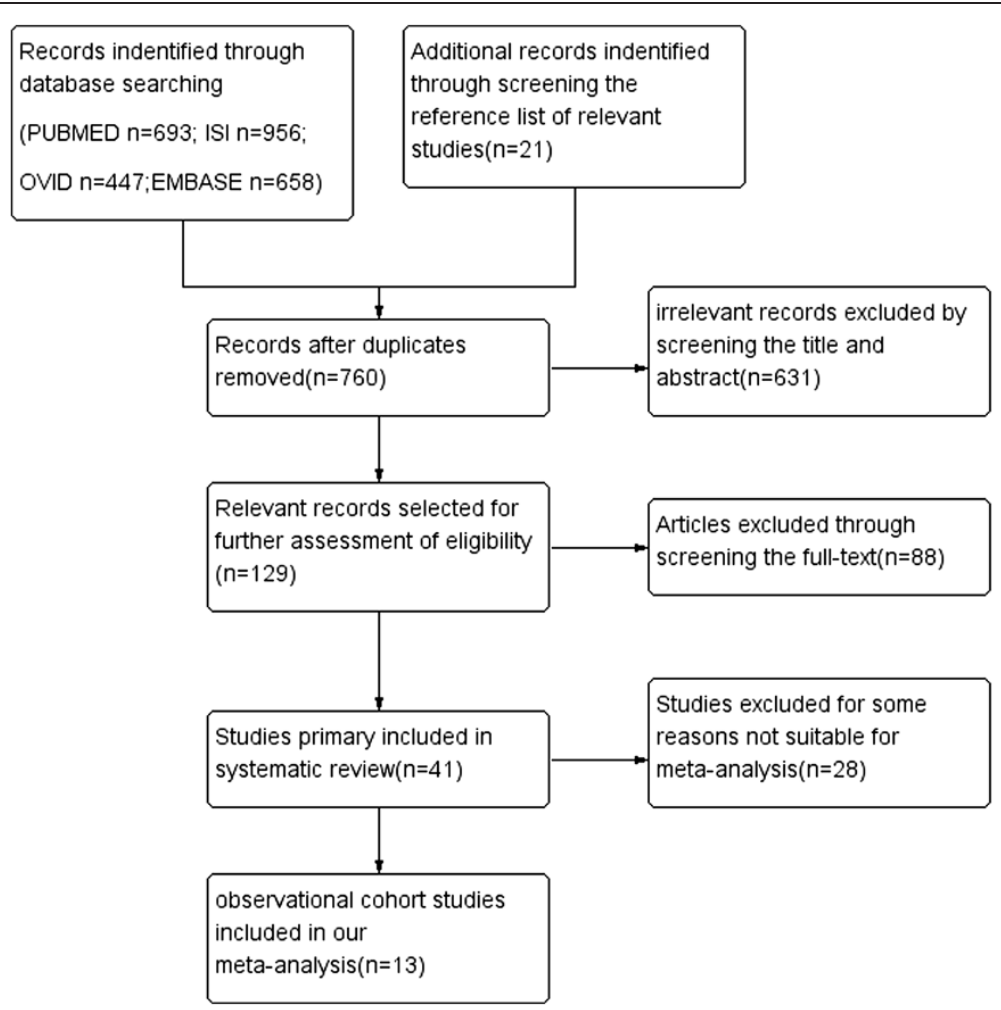

Figure 1 Study flow diagram of study selection. 
Table 1 Characteristics of studies reporting the relationship between hyperuricemia and CKD

\begin{tabular}{|c|c|c|c|c|c|c|c|c|c|c|}
\hline \multirow[t]{2}{*}{ Author/year } & \multirow[t]{2}{*}{ Country } & \multirow[t]{2}{*}{ Study type } & \multirow{2}{*}{$\begin{array}{l}\text { Follow-up } \\
\text { (mean) }\end{array}$} & \multirow[t]{2}{*}{ Source } & \multirow{2}{*}{$\begin{array}{l}\text { Selective criteria } \\
\text { for hyperuricaemia }\end{array}$} & \multirow{2}{*}{$\begin{array}{l}\text { Defination } \\
\text { of CKD }\end{array}$} & \multicolumn{2}{|l|}{ Events } & \multirow[t]{2}{*}{ AOR (AHR) $(95 \% \mathrm{Cl})$} & \multirow[t]{2}{*}{ Adjustments } \\
\hline & & & & & & & $\overline{\mathrm{HPU}}$ & Control & & \\
\hline $\begin{array}{l}\text { Ryoo et al. } \\
\text { [16] } 2013\end{array}$ & Korea & $\begin{array}{l}\text { Prospective } \\
\text { cohort }\end{array}$ & 4 years & $\mathrm{CP}$ & $>7.0 \mathrm{mg} / \mathrm{dL}$ & $\mathrm{eGFR}<60$ & $44 / 3910$ & $66 / 14868$ & $1.96(1.28-2.99)^{\#}$ & $\begin{array}{l}\text { Age, BMI, Diabetes, smoking, } \\
\text { alcohol, blood pressure, exercise, } \\
\text { hyperlipidaemia }\end{array}$ \\
\hline \multirow{2}{*}{$\begin{array}{l}\text { Chang et al. } \\
\text { [17] } 2013\end{array}$} & \multirow[t]{2}{*}{ Taiwan } & \multirow{2}{*}{$\begin{array}{l}\text { Prospective } \\
\text { cohort study }\end{array}$} & \multirow{2}{*}{$\begin{array}{l}4 \text { years } \\
\text { (>40 years old })\end{array}$} & \multirow[t]{2}{*}{$C P$} & \multirow{2}{*}{$\begin{array}{l}>7.5 \mathrm{mg} / \mathrm{dL} \text { (male) } \\
\text { and }>6.5 \mathrm{mg} / \mathrm{dL} \\
\text { (female) }\end{array}$} & \multirow[t]{2}{*}{$A C R>30$} & \multirow[t]{2}{*}{ N.R. } & \multirow[t]{2}{*}{ N.R. } & $1.42(1.27-1.59)^{\&}$ & \multirow{2}{*}{$\begin{array}{l}\text { Age, gender, BMI, Diabetes, } \\
\text { blood pressure, } \\
\text { Hypercholesteraemia, education }\end{array}$} \\
\hline & & & & & & & & & $3.54(2.11,5.94)^{\#}$ & \\
\hline \multirow[t]{2}{*}{$\begin{array}{l}\text { Zoppini et al. } \\
\text { [18] } 2012\end{array}$} & \multirow[t]{2}{*}{ Italy } & \multirow[t]{2}{*}{$\begin{array}{l}\text { Retrospective } \\
\text { cohort }\end{array}$} & \multirow[t]{2}{*}{5 years } & \multirow[t]{2}{*}{ DP } & \multirow[t]{2}{*}{$\begin{array}{l}>7.0 \mathrm{mg} / \mathrm{dL} \text { (male); } \\
>6.5 \mathrm{mg} / \mathrm{dL} \text { (female) }\end{array}$} & \multirow{2}{*}{$\begin{array}{l}\text { eGFR }<60 \\
\text { or macro- } \\
\text { albuminuria }\end{array}$} & \multirow{2}{*}{$47 / 159$} & \multirow[t]{2}{*}{$147 / 1290$} & $\begin{array}{l}1.20(1.03-1.57)^{\&} \\
2.01\end{array}$ & \multirow{2}{*}{$\begin{array}{l}\text { BMI, smoking, blood pressure, } \\
\text { albuminuria, duration of diabetes, } \\
\text { HbA1C }\end{array}$} \\
\hline & & & & & & & & & $(1.10-3.74)^{\#}$ & \\
\hline $\begin{array}{l}\text { Sonoda et al. } \\
\text { [19] } 2011\end{array}$ & Japan & $\begin{array}{l}\text { Prospective } \\
\text { cohort }\end{array}$ & $4-5$ years & $C P$ & $\begin{array}{l}>7.0 \mathrm{mg} / \mathrm{dL} \text { (male); } \\
>6.0 \mathrm{mg} / \mathrm{dL} \text { (female) }\end{array}$ & eGFR $<60$ & N.R. & N.R. & $1.09(1.01-1.18)^{\&}$ & BMI, BP, LDL, HDL, smoke, eGFR \\
\hline \multirow{2}{*}{$\begin{array}{l}\text { Kawashima et al. } \\
\text { [20] } 2011\end{array}$} & \multirow[t]{2}{*}{ Japan } & \multirow{2}{*}{$\begin{array}{l}\text { Retrospective } \\
\text { cohort }\end{array}$} & 95.2 & \multirow[t]{2}{*}{$C P$} & $>7.0 \mathrm{mg} / \mathrm{dL}$ & $\mathrm{eGFR}<60$ & $32 / 166$ & $68 / 1119$ & $3.99(2.59-6.15)^{\#}$ & Age, BMl, HDL, BP, blood \\
\hline & & & $( \pm 66.7)$ months & & & & & & & \\
\hline $\begin{array}{l}\text { Mok et al. } \\
\text { [21] } 2011\end{array}$ & Korea & $\begin{array}{l}\text { Severance } \\
\text { cohort }\end{array}$ & 6.5 years & $C P$ & $\begin{array}{l}>6.6 \text { mg/dL (male); } \\
>4.6 \mathrm{mg} / \mathrm{dL} \text { (female) }\end{array}$ & GFR $<60$ & $226 / 3450$ & $540 / 11489$ & $\begin{array}{l}2.1(1.6-2.9) \\
\text { male*; }\end{array}$ & $\begin{array}{l}\text { BMI, Diabetes, blood pressure, } \\
\text { Hypercholesteraemia }\end{array}$ \\
\hline & & & & & & & & & $\begin{array}{l}1.3(1.0-1.8) \\
\text { female* }^{*}\end{array}$ & \\
\hline $\begin{array}{l}\text { Yamada et al. } \\
\text { [22] } 2011\end{array}$ & Japan & $\begin{array}{l}\text { Retrospective } \\
\text { cohort }\end{array}$ & 5 years & $C P$ & $\begin{array}{l}>6.7 \mathrm{mg} / \mathrm{dL} \text { (male); } \\
>4.8 \mathrm{mg} / \mathrm{dL} \text { (female) }\end{array}$ & eGFR $<60$ & $343 / 3119$ & $282 / 11280$ & $\begin{array}{l}1.42(1.28-1.58) \\
\text { male\&; }\end{array}$ & $\begin{array}{l}\text { Age, BMl, Diabetes, smoking, } \\
\text { alcohol, blood pressure, }\end{array}$ \\
\hline & & & & & & & & & $\begin{array}{l}1.32(1.12-1.56) \\
\text { female\& }\end{array}$ & albuminuria, hyperlipidaemia \\
\hline $\begin{array}{l}\text { Wang et al. } \\
\text { [23] } 2011\end{array}$ & China & $\begin{array}{l}\text { Prospective } \\
\text { cohort }\end{array}$ & 3 years & $\mathrm{CP}$ & $\begin{array}{l}>7.0 \mathrm{mg} / \mathrm{dL} \text { (male); } \\
>6.0 \mathrm{mg} / \mathrm{dL} \text { (female) }\end{array}$ & GFR $<60$ & N.R. & N.R. & $1.03(1.01-1.06)^{\&}$ & $\begin{array}{l}\text { Age, gender, BMl, smoking, } \\
\text { alcohol, exercise, } \\
\text { Hypercholesteraemia, education, } \\
\text { hyperlipidaemia }\end{array}$ \\
\hline $\begin{array}{l}\text { Jalal et al. } \\
\text { [24] } 2010\end{array}$ & America & $\begin{array}{l}\text { Prospective } \\
\text { observational } \\
\text { study. }\end{array}$ & 6 years & DP & N.R. & $A C R>30$ & N.R. & N.R. & $1.80(1.20-2.80)^{\#}$ & $\begin{array}{l}\text { Age, gender, BMl, blood pressure, } \\
\text { albuminuria, duration of diabetes, } \\
\text { HbA1c, serum creatinine, } \\
\text { medication for CKD or } \\
\text { hyperuricaemia }\end{array}$ \\
\hline Yen et al. & Taiwan & Prospective & 32.4 months & $\mathrm{CP}$ & $>6.6 \mathrm{mg} / \mathrm{dL}$ & $e G F R<60$ & $84 / 312$ & $60 / 488$ & $0.997(0.847-1.175)^{\&}$ & Age, gender, BMI, Diabetes, \\
\hline & & & & $\begin{array}{l}\text { (>65 years } \\
\text { old) }\end{array}$ & & & & & & $\begin{array}{l}\text { smoking, blood pressure, } \\
\text { Hypercholesteraemia, } \\
\text { albuminuria, serum creatinine }\end{array}$ \\
\hline $\begin{array}{l}\text { Weiner et al. } \\
\text { [26] } 2008\end{array}$ & America & $\begin{array}{l}\text { Prospective } \\
\text { cohort }\end{array}$ & 8.5 years & $\mathrm{CP}$ & $\begin{array}{l}>7.4 \mathrm{mg} / \mathrm{dL} \text { (male); } \\
>6.1 \mathrm{mg} / \mathrm{dl} \text { (female) }\end{array}$ & eGFR $<60$ & $260 / 3167$ & $481 / 10171$ & $1.07(1.01-1.14)^{\&}$ & $\begin{array}{l}\text { Age, gender, race, diabetes, } \mathrm{BP}, \\
\text { cardiac disease, smoke, alcohol } \\
\text { use, education, lipid, albumin }\end{array}$ \\
\hline
\end{tabular}


Table 1 Characteristics of studies reporting the relationship between hyperuricemia and CKD (Continued)

\begin{tabular}{|c|c|c|c|c|c|c|c|c|c|c|}
\hline $\begin{array}{l}\text { Obermayr et al. } \\
\text { [27] } 2008\end{array}$ & Austria & $\begin{array}{l}\text { Retrospective } \\
\text { cohort }\end{array}$ & 7 years & $C P$ & $7.0-8.9$ mg/dL & $e G F R<60$ & N.R. & N.R. & $1.26(1.02-1.55)^{8}$ & $\begin{array}{l}\text { Age, gender, Diabetes, LDL, } \\
\text { hyperlipidaemia, medication for } \\
\text { CKD or hyperuricaemia }\end{array}$ \\
\hline $\begin{array}{l}\text { Domrongkitchaiporn } \\
\text { et al. [28] } 2005\end{array}$ & Thailand & $\begin{array}{l}\text { Retrospective } \\
\text { cohort }\end{array}$ & 12 years & $C P$ & $6.30-14.50 \mathrm{mg} / \mathrm{dL}$ & eGFR $<60$ & N.R. & N.R. & $1.82(1.12-2.98)^{\#}$ & $\begin{array}{l}\text { BMI, Diabetes, smoking, blood } \\
\text { pressure, Hypercholesteraemia, } \\
\text { albuminuria }\end{array}$ \\
\hline
\end{tabular}

${ }^{8} \mathrm{AOR}$ calculated using uric acid level for incidence of onset of CKD; ${ }^{\mathrm{A} A O R}$ calculated using hyperuricemia individuals compared with normal individuals for new-onset $\mathrm{CKD} ;{ }^{*} \mathrm{AOR}$ calculated using individuals of the last uric acid quartiles compared with first uric acid quartiles for the risk for CKD; N.R.: not reported; eGFR $<60$ means eGFR $<60 \mathrm{~mL} / \mathrm{min}^{\prime} 1.73 \mathrm{~m}$; ACR $>30 \mathrm{means}$ urinary albumin-to-creatinine ratio $>30 \mathrm{mg} / \mathrm{g}$; HPU: hyperuricemia; CP: community-based population; DP: patients with diabetes mellitus. 
Different cut-off levels were used for defining hyperuricemia (Table 1). An SUA level $>7.0 \mathrm{mg} / \mathrm{dL}$ in men or $>6.0 \mathrm{mg} / \mathrm{dL}$ in women was used in the majority of studies.

Adjustments made for potential confounders of at least three factors were mentioned in all 13 studies.

Serum uric acid level and development of new-onset CKD Seven studies provided the adjusted OR of increasing risk of new-onset CKD per $1 \mathrm{mg} / \mathrm{dL}$ SUA elevated. The results of pooled analysis revealed a significant positive association between SUA and incidence of CKD (summary OR, 1.06; 95\% CI, 1.04-1.08) with evidence of significant heterogeneity among studies $\left(Q=34.76, P<0.001, I^{2}=83 \%\right)$ (Figure 2).

On examining study variability using meta-regression analysis, we found that the population age was the cause of heterogeneity. In one study that used an elderly population [17], the OR was comparatively higher than the other studies. By excluding this particular study, we observed that a clear positive association was retained (summary OR, 1.05; 95\% CI, 1.02-1.07) while heterogeneity was greatly reduced $\left(Q=6.93, P=0.23, I^{2}=28 \%\right)$ (Figure 3).

\section{Hyperuricemia and development of new-onset CKD}

Six studies provided risk ratios for the association between hyperuricemia and new-onset CKD in non-CKD populations during follow-up. Results of summary analysis of these six studies showed a significant association between hyperuricemia and increasing risk of the incidence of
CKD (summary OR, 2.59; 95\% CI, 2.14-3.13) in a random effects model. Moderate heterogeneity was found among studies $\left(Q=9.55 ; P=0.09 ; I^{2}=61.2 \%\right)$ (Figure 4$)$.

Results of meta-regression analysis indicated that heterogeneity was caused by follow-up year and sex proportion. One study from Japan only included male volunteers and had a follow-up of 18 years and reported a comparatively higher OR and contributed $44 \%$ of heterogeneity [20]. After excluding this study, no significant heterogeneity across the remaining studies was detected $\left(Q=0.480, P=0.31, I^{2}=17 \%\right)$. For these five other studies, the association between hyperuricemia and the onset of CKD was still significantly positive (summary OR, 2.11; 95\% CI, 1.70-2.61).

\section{Subgroup analysis}

Results of subgroup analysis according to sources of participants, geographical area, sex and follow-up year are shown in Table 2.

For subgroup analysis for source of participants, no differences were observed in summary OR for associations between hyperuricemia and CKD in patients with diabetes and the community-based population (test for subgroup difference, $P=0.07$ ). Both groups showed significant independent associations between hyperuricemia and new-onset CKD.

For geographical location, positive results were observed between SUA and the development of new-onset CKD in both Asian countries (summary OR, 1.05; 95\% CI, 1.03-1.08) and Western countries (summary OR, 1.17; $95 \%$ CI, 1.11-1.22).

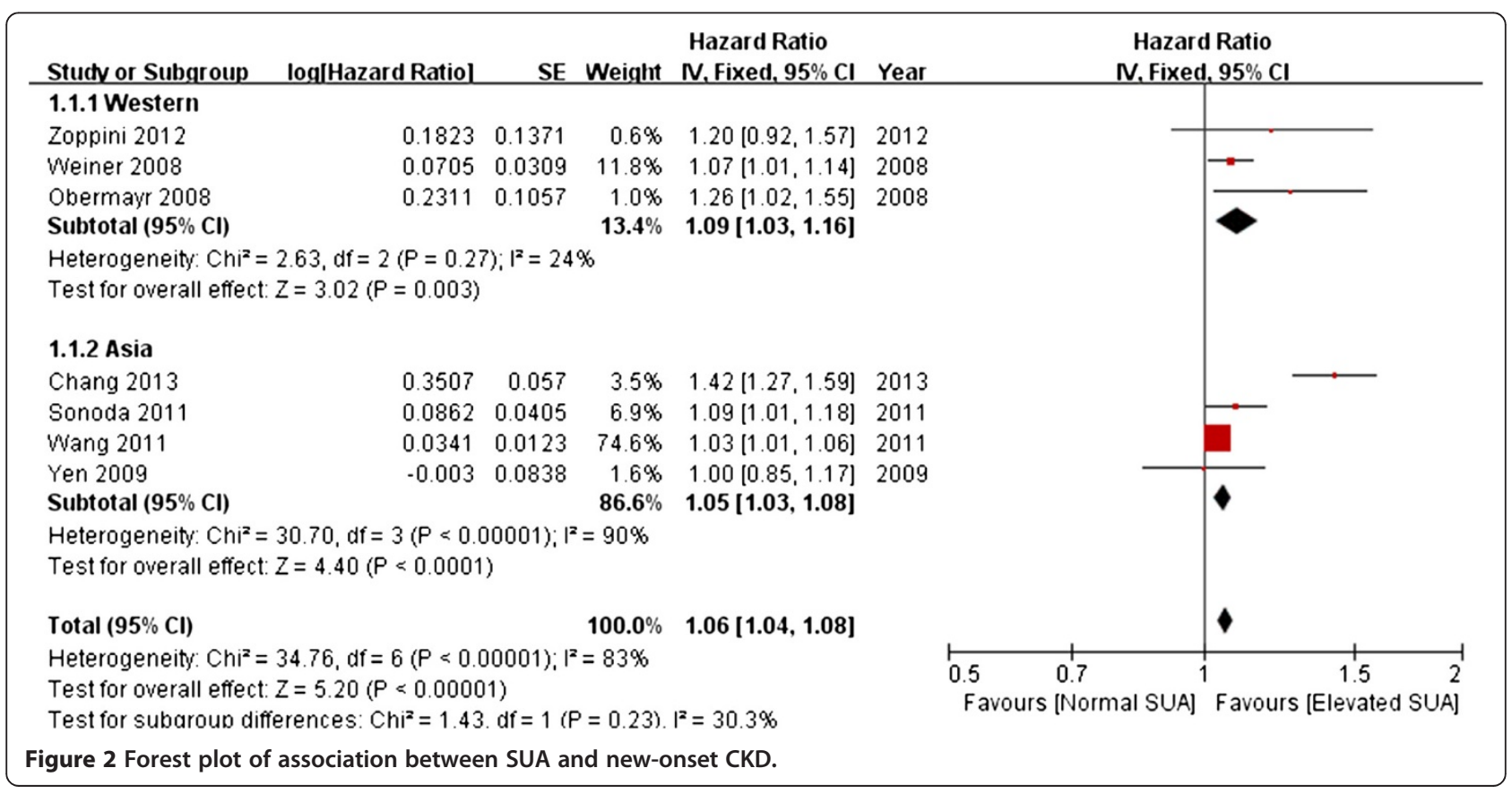




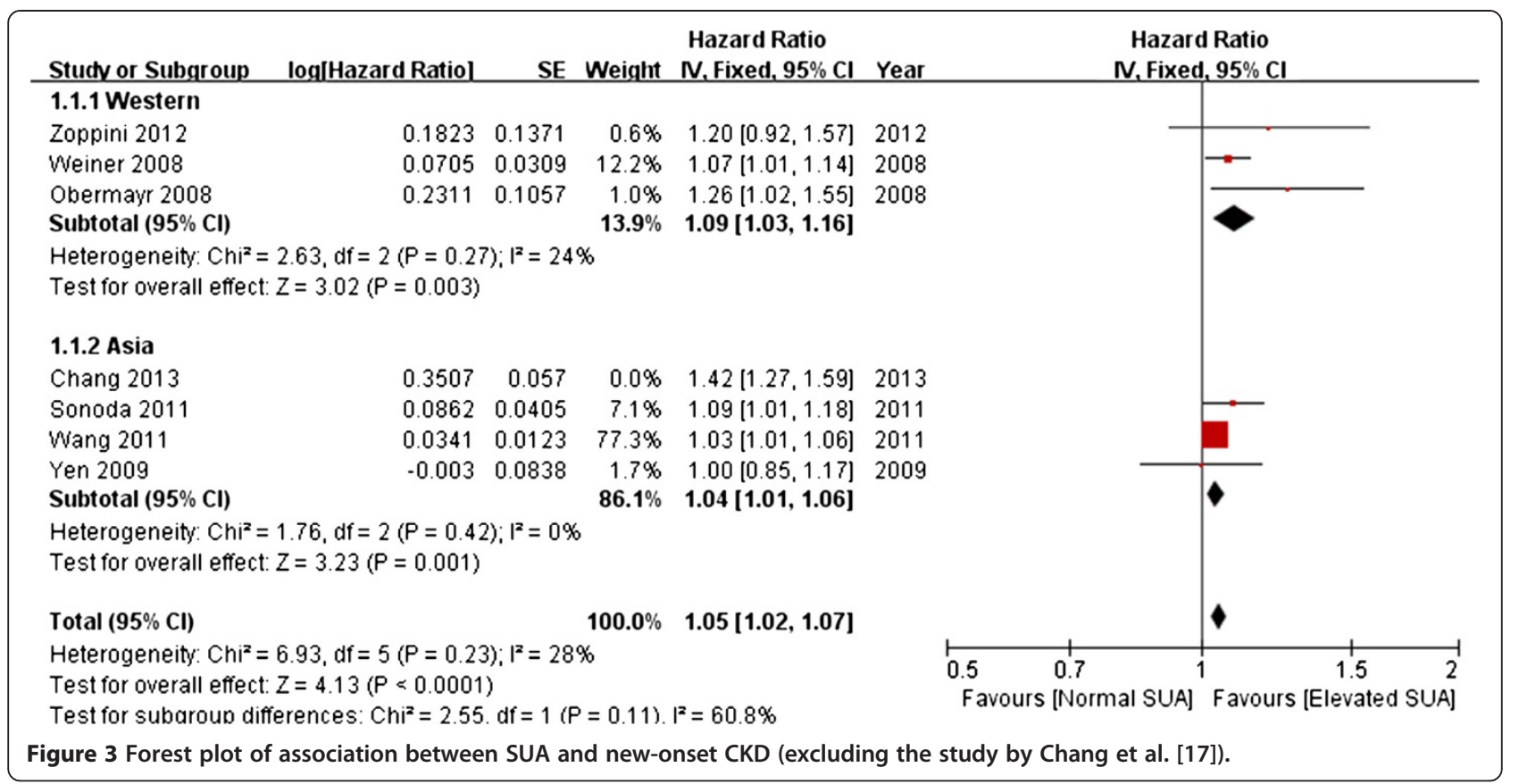

Three studies provided results for males and females, and the results of pooled analysis by sex indicated a similar significant positive association between hyperuricemia and new-onset CKD in both males (summary OR, 1.43; 95\% CI, 1.05-1.94.) and females (summary OR, 1.21; 95\% CI, 1.04-1.41) (Figure 5). A study by Mok et al. reported that a stronger association beween hyperuricemia and CKD for in females than males, a finding contrary to results from the other studies, contributed to the heterogeneity of pooled analysis [21].
The subgroup of follow-up year revealed that the longer time period for observation, the greater the risk of the development of new-onset CKD in patients with elevated uric acid levels.

\section{Publication bias}

Funnel plots revealed no evidence of publication bias for associations between increasing SUA and new-onset CKD and the relationship between hyperuricemia and newonset CKD (Figure 6a, Figure 6b). Results of the Begg

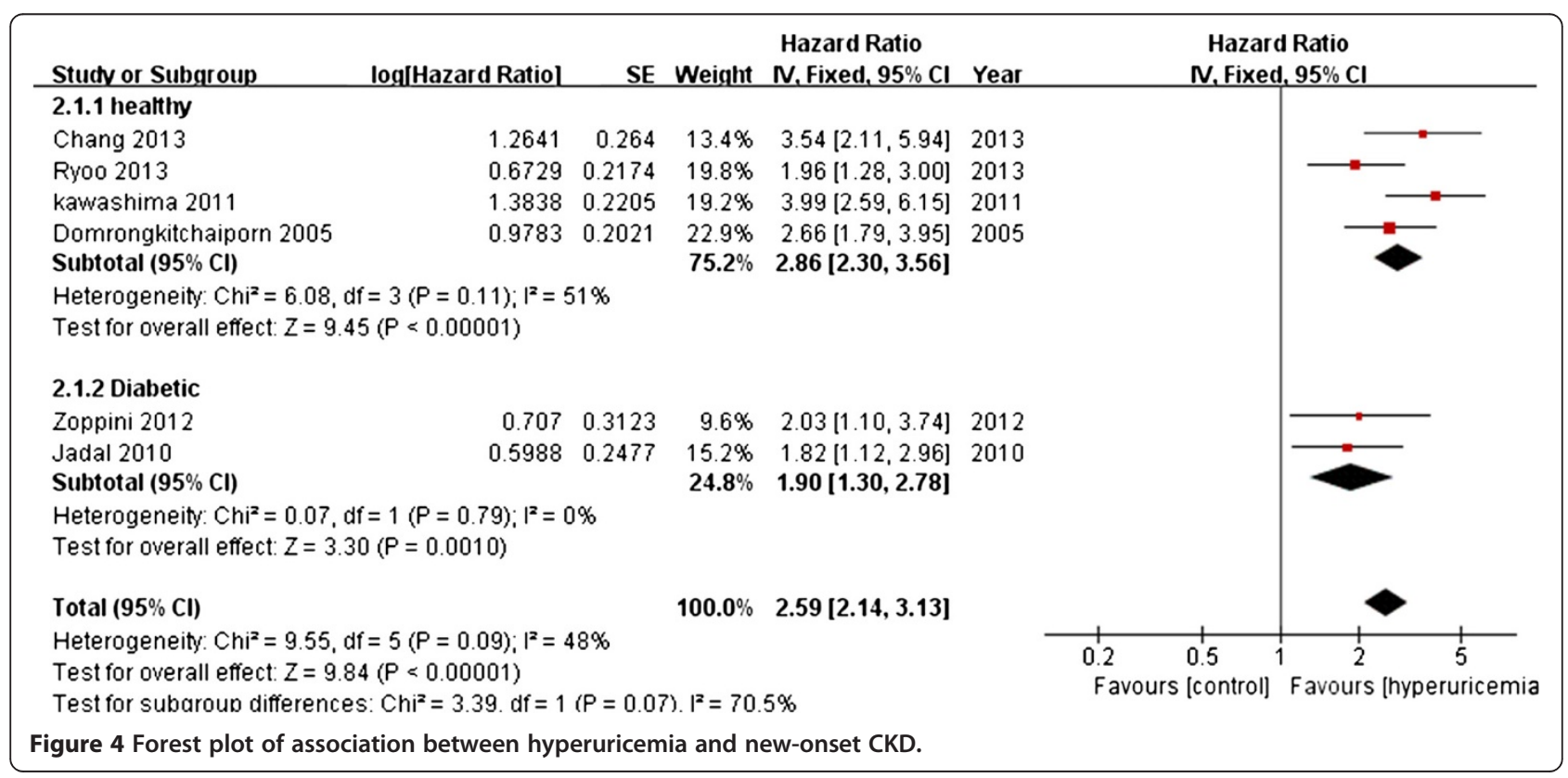


Table 2 Summary of relative risks for associations between SUA and development of CKD

\begin{tabular}{|c|c|c|c|c|c|}
\hline \multirow[t]{2}{*}{ Subgroup } & \multirow[t]{2}{*}{ Reference } & \multirow{2}{*}{$\begin{array}{l}\text { SOR } \\
(95 \% \mathrm{Cl})\end{array}$} & \multicolumn{3}{|c|}{ Tests for heterogeneity } \\
\hline & & & $\bar{Q}$ & $P$ & $I^{2}(\%)$ \\
\hline \multicolumn{6}{|c|}{ Geographic region } \\
\hline Asia & {$[17,19,23,25]$} & $1.05(1.03-1.08)^{\triangle}$ & 30.7 & $<0.001^{*}$ & 90 \\
\hline Asia $^{\#}$ & {$[19,23,25]$} & $1.04(1.01-1.06)^{\Delta}$ & 1.76 & 0.42 & 0.00 \\
\hline Western & {$[18,26,27]$} & $1.17(1.11-1.22)^{\Delta}$ & 0.64 & 0.724 & 0.00 \\
\hline \multicolumn{6}{|l|}{ Population } \\
\hline Healthy people & {$[16,17,20,28]$} & $2.59(2.07-3.23)^{\boldsymbol{\Lambda}}$ & 6.08 & $0.02^{*}$ & 69 \\
\hline Diabetic & {$[18,24]$} & $1.90(1.30-2.78)^{\wedge}$ & 0.07 & 0.79 & 0 \\
\hline \multicolumn{6}{|l|}{ Sex } \\
\hline Male & {$[21,22,26]$} & $1.43(1.05-1.94)^{\Delta}$ & 32.98 & $0.001^{*}$ & 94 \\
\hline Female & {$[21,22,26]$} & $1.21(1.04-1.41)^{\Delta}$ & 4.57 & 0.07 & 63 \\
\hline \multicolumn{6}{|l|}{ Follow-up } \\
\hline \multirow[t]{2}{*}{$<5$ years } & {$[2,19,23,25]$} & $1.03(1.01-1.06)^{\Delta}$ & 0.19 & 0.66 & 0 \\
\hline & {$[16,17]$} & $2.49(1.79-3.46)^{\boldsymbol{\Lambda}}$ & 2.99 & 0.08 & 67 \\
\hline \multirow[t]{2}{*}{$\geq 5$ years } & {$[17,18,26,27]$} & $1.09(1.04-1.14)^{\Delta}$ & 2.63 & 0.45 & 0 \\
\hline & {$[18,20,24,28]$} & $2.64(2.09-3.32)^{\wedge}$ & 6.48 & 0.09 & 54 \\
\hline
\end{tabular}

SOR: summary odds ratio; ${ }^{\#}$ Studies in Asian areas (except study by Chang et al. [17]); ${ }^{\circ}$ OR calculated using continuous variables; per 1 mg/dL increase of uric acid levels; ${ }^{\triangle}$ OR calculated using dichotomous variables; hyperuricemia compared with normal; *heterogeneity exists.

adjusted rank correlation test for detecting publication bias were negative (SUA, $P=0.576$; hyperuricemia, 0.452). The Egger regression asymmetry test showed no significance for either SUA $(P=0.176)$ or hyperuricemia $(P=0.977)$.

\section{Discussion}

A previous meta-analysis conducted by Li et al. reported that uric acid level was independently associated with renal disease; however, the researchers included results from patients with end-stage renal disease or acute renal impairment and patients who received dialysis [29]. Additionally, the definition of renal dysfunction used by Li et al. did not follow the KDOQI CKD guidelines [12].

The present meta-analysis and systematic review found that elevated SUA was significantly positively associated with new-onset CKD in populations with normal renal function: individuals with hyperuricemia had an increased

\begin{tabular}{|c|c|c|c|c|c|c|c|}
\hline Study or Subgroup & log[Hazard Ratio] & SE & Weight & $\begin{array}{c}\text { Hazard Ratio } \\
\text { N, Random, } 95 \% \mathrm{Cl}\end{array}$ & Year & $\begin{array}{r}\text { Hazar } \\
N, \text { Rando } \\
\end{array}$ & $\begin{array}{l}\text { d Ratio } \\
\text { m, 95\% Cl }\end{array}$ \\
\hline \multicolumn{8}{|l|}{ 3.1.1 Male } \\
\hline Yamada 2011 & 0.3507 & 0.053 & $16.9 \%$ & $1.42[1.28,1.58]$ & 2011 & & $\rightarrow$ \\
\hline Mok 2011 & 0.2779 & 0.0617 & $15.9 \%$ & $1.32[1.17,1.49]$ & 2011 & & \\
\hline Weiner 2008 & 0.0488 & 0.0457 & $17.7 \%$ & $1.05[0.96,1.15]$ & 2008 & & \\
\hline Subtotal $(95 \% \mathrm{Cl})$ & & & $50.6 \%$ & $1.25[1.03,1.52]$ & & & \\
\hline \multicolumn{8}{|c|}{$\begin{array}{l}\text { Heterogeneity: } \operatorname{Tau}^{2}=0.03 ; \mathrm{Chi}^{2}=20.62, d f=2(P<0.0001) ; \mathrm{I}^{2}=90 \% \\
\text { Test for overall effect } Z=2.27(P=0.02)\end{array}$} \\
\hline \multicolumn{8}{|l|}{ 3.1.2 Female } \\
\hline Yamada 2011 & 0.2776 & 0.0838 & $13.4 \%$ & $1.32[1.12,1.56]$ & 2011 & & \\
\hline Mok 2011 & 0.0912 & 0.0465 & $17.6 \%$ & $1.10[1.00,1.20]$ & 2011 & & 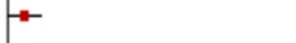 \\
\hline Weiner 2008 & 0.0877 & 0.0397 & $18.3 \%$ & $1.09[1.01,1.18]$ & 2008 & & $=$ \\
\hline Subtotal $(95 \% \mathrm{Cl})$ & & & $\mathbf{4 9 . 4} \%$ & $1.14[1.04,1.24]$ & & & \\
\hline \multicolumn{8}{|c|}{$\begin{array}{l}\text { Heterogeneity: } \operatorname{Tau}^{2}=0.00 ; \mathrm{Chi}^{2}=4.48, d f=2(P=0.11) ; \mathrm{I}^{2}=55 \% \\
\text { Test for overall effect } Z=2.78(P=0.006)\end{array}$} \\
\hline \multirow{2}{*}{\multicolumn{6}{|c|}{ 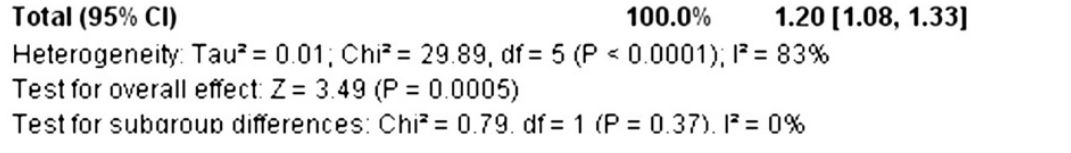 }} & & \\
\hline & & & & & & 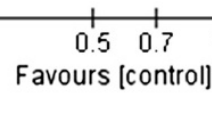 & 11.52 \\
\hline igure 5 Forest plot o & association between & SUA and & d new-on & set CKD in differen & end & & \\
\hline
\end{tabular}



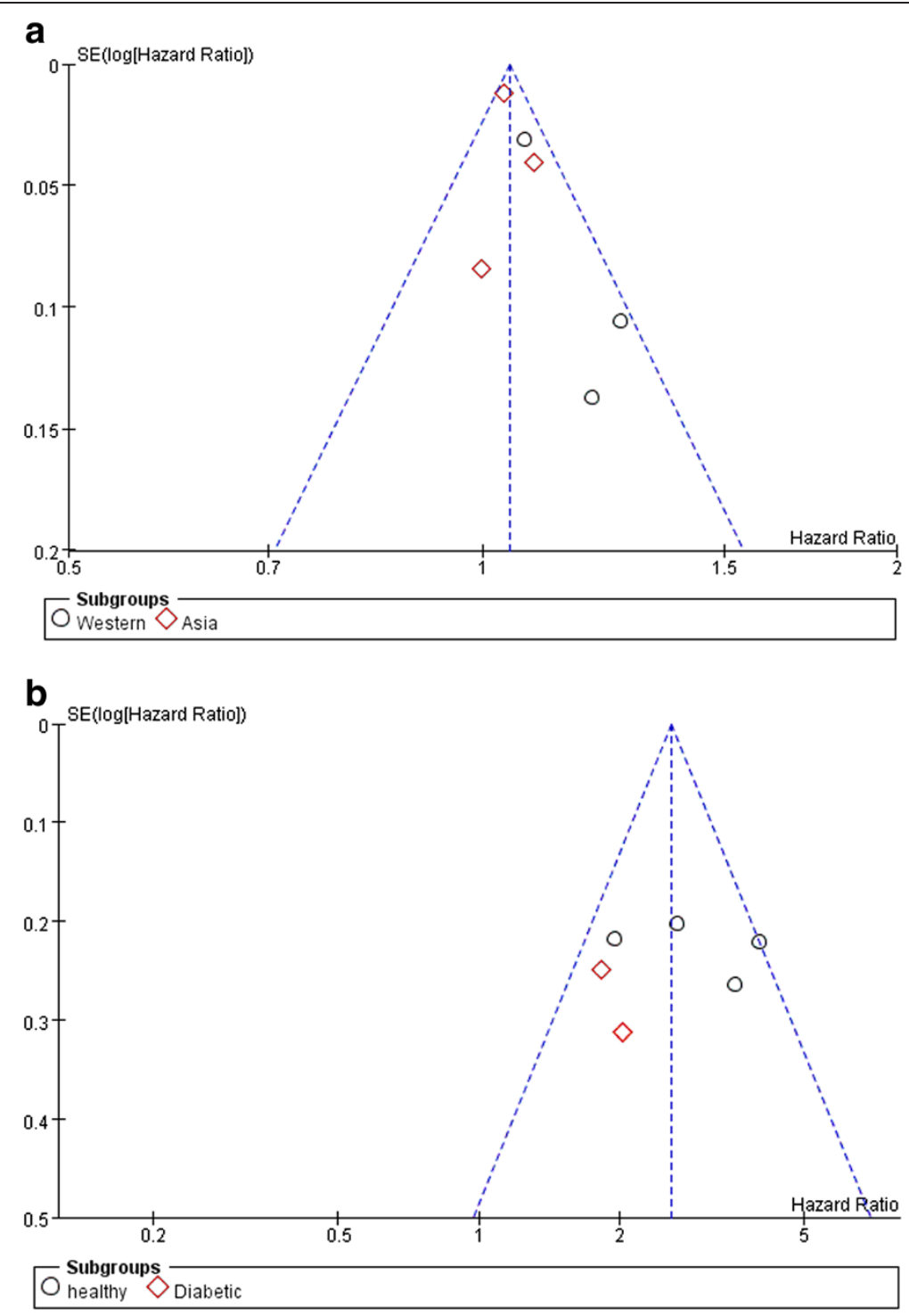

Figure 6 Funnel plot of association between increasing SUA or hyperuricemia and new-onset CKD. a. Funnel plot of association between increasing SUA and new-onset CKD, b. Funnel plot of association between hyperuricemia and new-onset CKD.

risk of new-onset CKD compared with those without hyperuricemia. Although the adjusted OR for the association between elevated SUA and the development of new-onset CKD was not great, it was found to be independent of sex, age, body mass index, alcohol intake, smoking, hypertension, metabolic syndrome, hypertriglyceridemia, diabetes and medication. In subgroup analysis, we found that the summary OR for the association between elevated SUA and development of new-onset CKD increased with increasing length of follow-up time, indicating hyperuricemia may play a role in the long-term progression of renal function. With regard to geographic distribution, subgroup analysis showed a stronger association between elevated SUA and CKD development in Western countries compared with Asian populations. This difference may be because of race, geographical or diet differences, as the Western diet might contain more purine-rich foods. Two studies by Chen and Xue et al. reported that the strength of the association between hyperuricemia and CKD was greater in males than in females [30,31]. However, the results of our subgroup analysis found that males and females had similar risks of CKD and new-onset CKD associated with hyperuricemia. High-quality etiological studies are therefore needed.

Our study is of significant clinical and public health importance. CKD is a very common disease and its prevalence is increasing in both developed and developing countries. Treating hyperuricemia may delay or even prevent the onset of CKD. Clinical studies conducted by Feig et al. suggested that treating hyperuricemia in 
adolescents with newly diagnosed hypertension was effective at lowering blood pressure, which indirectly decreased the risk of incidence of CKD [32]. A case-control study conducted by Kanbay et al. revealed that allopurinol intervention in individuals with eGFR $>60 \mathrm{~mL} / \mathrm{min} / 1.73 \mathrm{~m}^{2}$ significantly increased eGFR at follow-up [33]. A recent meta-analysis by Bose et al. reported that uric acid-lowering therapy with allopurinol could retard the progression of CKD, but had no effect on proteinuria and blood pressure [34]. A significant association between metabolic syndrome and CKD has been reported in several clinical studies and in one meta-analysis [35].

The present meta-analysis has potential limitations. First, observational cohort studies may have unknown confounding attribution bias and selection bias, which may distort the true effects. Second, OR or HR calculated using continuous and dichotomous variables were pooled to reduce heterogeneity. Some heterogeneity caused by different statistical methods is inevitable; for example, heterogeneity for pooled analysis in males was caused by one study using uric acid per SD for their calculations. Third, confounding variables are likely to be present. Hyperuricemia may also reflect an unhealthy lifestyle, such as smoking, obesity and hyperlipidemia. These risk factors have been confirmed to play a pathogenic role in diabetes, hypertension and metabolic syndrome, which also contribute to renal dysfunction. Half the studies examined did not make adjustments for metabolic syndrome and diabetes in their calculations. Fourth, heterogeneity of cumulative analysis, which may be caused by population source, follow-up year and gender, could only be partially explained by subgroup analysis on examining these factors. Subgroup analysis by race and different stages of CKD was not possible because of a lack of data. Finally, as the publication bias test was statistical, it is possible for publication bias to exist as the meta-analysis can only use the published studies.

As with any observational study, associations do not imply causality. For example, in a recent Mendelian randomization analysis of two large cohorts, though estimates confirmed known observational associations between plasma uric acid and hyperuricemia with risk of ischemic heart disease, when using genotypic instruments for uric acid and hyperuricemia, the author saw no evidence for causal associations between uric acid and ischemic heart disease [36]. Similarly, the association found with our meta-analysis does not necessarily mean a causal relationship between SUA and CKD. However, our results were consistent with biologically plausible explanations mentioned in other studies.

Traditionally, gout is associated with renal disease while hyperuricemia results in intraluminal crystals in collecting ducts of nephrons [37]. Uric acid crystals adhere to the surface of renal epithelial cells and induce an acute inflammatory response [38]. Other than the uric acid crystal effect, hyperuricemia has been reported to cause an afferent renal arteriopathy and tubulointerstitial fibrosis in the kidney by activating the renin-angiotensin-aldosterone system [39]. It has also been shown that hyperuricemia activates cytoplasmic phospholipase A2 and inflammatory transcription factor nuclear factor kappa- $\beta$, which inhibit proximal tubular proliferation [40]. Furthermore, an elevated SUA level accelerates renal injury progression in the remnant kidney model via a mechanism linked to high systemic blood pressure and cyclooxygenase-2-mediated, thromboxane-induced vascular disease [41]. In rat experiments conducted by Ryu et al., uric acid induced phenotypic transition of cultured renal tubular cells, as epithelial-to-mesenchymal transition, which is a known mechanism for renal fibrosis [42].

We found that hyperuricemia could be a risk factor for CKD. For patients with subclinical kidney disease, hyperuricemia can be the consequence of decreased renal uric acid excretion, which could in turn further exacerbate kidney function. Therefore, the causal relationship between hyperuricemia and CKD is far more complicated than a simple cause-and-effect relationship. This means that the results of the current study should be interpreted with caution.

Regardless of whether elevated SUA is solely a marker of subclinical chronic renal dysfunction or an independent risk factor for the development of CKD, careful attention is warranted. Our study emphasizes the need to identify individuals with hyperuricemia, as early intervention that decreases SUA levels may lower the risk of developing CKD. Randomized controlled studies of high quality are required to investigate the effects of decreasing uric acid levels in CKD patients and in individuals at high risk of developing CKD.

\section{Conclusions}

This systematic review and meta-analysis demonstrated that, in the long-term follow-up of non-CKD individuals, elevated SUA levels showed an increased risk of developing chronic renal dysfunction.

\section{Additional files}

\section{Additional file 1: The MOOSE Checklist of the article. \\ Additional file 2: The NOS quality assessment of the included} cohort studies.

\section{Abbreviations}

CKD: Chronic kidney disease; KDOQI: National kidney foundation kidney disease outcomes and quality initiative; NOS: Newcastle-ottawa scale.

\section{Competing interests}

The authors declare that they have no competing interests. 


\section{Authors' contributions}

PF conceived the study and participated in its design. LL and CY participated in the design of the study and performed statistical analyses. The manuscript was prepared by $L L, C Y, Y Z, X Z, F L$ and PF. All authors participated in discussions about the manuscript and approved the final version.

\section{Acknowledgments}

We gratefully acknowledge the grant provided by Songmin Huang, Sheyu Li and Xi Tang for writing assistance for this study.

\section{Author details}

${ }^{1}$ Division of Nephrology, West China Hospital of Sichuan University, Chengdu 610041, Sichuan, China. ${ }^{2}$ Division of General Surgery, West China Hospital of Sichuan University, Chengdu 610041, Sichuan, China.

Received: 15 January 2014 Accepted: 18 July 2014

Published: 27 July 2014

\section{References}

1. Levey AS, Atkins R, Coresh J, Cohen EP, Collins AJ, Eckardt KU, Nahas ME, Jaber BL, Jadoul M, Levin A, Powe NR, Rossert J, Wheeler DC, Lameire N, Eknoyan G: Chronic kidney disease as a global public health problem: approaches and initiatives - a position statement from kidney disease improving global outcomes. Kidney Int 2007, 72:247-259.

2. Tanner RM, Brown TM, Muntner P: Epidemiology of obesity, the metabolic syndrome, and chronic kidney disease. Curr Hypertens Rep 2012, 14:152-159.

3. Pyram R, Kansara A, Banerji MA, Loney-Hutchinson L: Chronic kidney disease and diabetes. Maturitas 2012, 71:94-103.

4. Levin A: Identification of patients and risk factors in chronic kidney disease-evaluating risk factors and therapeutic strategies. Nephrol Dial Transplant 2001, 16(Suppl 7):57-60.

5. Becker MA, Jolly M: Hyperuricemia and associated diseases. Rheum Dis Clin North Am 2006, 32:275-293. v-vi.

6. Kang DH, Han L, Ouyang X, Kahn AM, Kanellis J, Li P, Feng L, Nakagawa T, Watanabe S, Hosoyamada M, Endou H, Lipkowitz M, Abramson R, Mu W, Johnson RJ: Uric acid causes vascular smooth muscle cell proliferation by entering cells via a functional urate transporter. Am J Nephrol 2005, 25:425-433.

7. Reungjui S, Roncal CA, Mu W, Srinivas TR, Sirivongs D, Johnson RJ, Nakagawa T: Thiazide diuretics exacerbate fructose-induced metabolic syndrome. J Am Soc Nephrol 2007, 18:2724-2731.

8. Kang DH, Park SK, Lee IK, Johnson RJ: Uric acid-induced C-reactive protein expression: implication on cell proliferation and nitric oxide production of human vascular cells. J Am Soc Nephrol 2005, 16:3553-3562.

9. Ford ES, Li C, Cook S, Choi HK: Serum concentrations of uric acid and the metabolic syndrome among US children and adolescents. Circulation 2007, 115:2526-2532.

10. Kang DH, Chen W: Uric acid and chronic kidney disease: new understanding of an old problem. Semin Nephrol 2011, 31:447-452.

11. Jalal DI, Chonchol M, Chen W, Targher G: Uric acid as a target of therapy in CKD. Am J Kidney Dis 2013, 61:134-146.

12. KDOQ/ Clinical Practice Guidelines for Chronic Kidney Disease: Evaluation, Classification, and Stratification. National Kindey Foundation; 2000. http://www.kidney.org/professionals/KDOQI/guideline.

13. Wells G, Shea B, O'connell D, Petersen J, Welch V, Losos M, Tugwell P: The Newcastle-Ottawa Scale (NOS) for assessing the quality of nonrandomised studies in meta-analyses. Ottawa Health Research Institute. http://www.ohri. ca/programs/clinical_epidemiology/oxfordas.

14. Green JPHS: Cochrane Handbook for Systematic Reviews of Interventions. 2011. http://www.cochrane.org/training/cochrane-handbook.

15. Stroup DF, Berlin JA, Morton SC, Olkin I, Williamson GD, Rennie D, Moher D, Becker BJ, Sipe TA, Thacker SB: Meta-analysis of observational studies in epidemiology: a proposal for reporting. Meta-analysis Of Observational Studies in Epidemiology (MOOSE) group. JAMA 2000, 283:2008-2012.

16. Ryoo JH, Choi JM, Oh CM, Kim MG: The association between uric acid and chronic kidney disease in Korean men: a 4-year follow-up study. J Korean Med Sci 2013, 28:855-860.

17. Chang HY, Lee PH, Lei CC, Tung CW, Hsu YC, Huang TJ, Lu LC, Lin CL: Hyperuricemia is an independent risk factor for new onset micro-albuminuria in a middle-aged and elderly population: a prospective cohort study in taiwan. PLoS One 2013, 8:e61450.

18. Zoppini G, Targher G, Chonchol M, Ortalda V, Abaterusso C, Pichiri I, Negri C, Bonora E: Serum uric Acid levels and incident chronic kidney disease in patients with type 2 diabetes and preserved kidney function. Diabetes Care 2012, 35:99-104.

19. Sonoda H, Takase H, Dohi Y, Kimura G: Uric acid levels predict future development of chronic kidney disease. Am J Nephrol 2011, 33:352-357.

20. Kawashima M, Wada K, Ohta H, Terawaki H, Aizawa Y: Association between asymptomatic hyperuricemia and new-onset chronic kidney disease in Japanese male workers: a long-term retrospective cohort study. BMC Nephrol 2011, 12:31.

21. Mok Y, Lee SJ, Kim MS, Cui W, Moon YM, Jee SH: Serum uric acid and chronic kidney disease: the Severance cohort study. Nephrol Dial Transplant 2012, 27:1831-1835.

22. Yamada T, Fukatsu M, Suzuki S, Wada T, Joh T: Elevated serum uric acid predicts chronic kidney disease. Am J Med Sci 2011, 342:461-466.

23. Wang S, Shu Z, Tao Q, Yu C, Zhan S, Li L: Uric acid and incident chronic kidney disease in a large health check-up population in Taiwan. Nephrology (Carlton) 2011, 16:767-776.

24. Jalal DI, Rivard CJ, Johnson RJ, Maahs DM, McFann K, Rewers M, Snell-Bergeon JK Serum uric acid levels predict the development of albuminuria over 6 years in patients with type 1 diabetes: findings from the Coronary Artery Calcification in Type 1 Diabetes study. Nephrol Dial Transplant 2010, 25:1865-1869.

25. Yen CJ, Chiang CK, Ho LC, Hsu SH, Hung KY, Wu KD, Tsai TJ: Hyperuricemia associated with rapid renal function decline in elderly Taiwanese subjects. J Formos Med Assoc 2009, 108:921-928.

26. Weiner DE, Tighiouart H, Elsayed EF, Griffith $J$ L, Salem DN, Levey AS: Uric acid and incident kidney disease in the community. J Am Soc Nephrol 2008, 19:1204-1211.

27. Obermayr RP, Temml C, Knechtelsdorfer M, Gutjahr G, Kletzmayr J, Heiss S, Ponholzer A, Madersbacher S, Oberbauer R, Klauser-Braun R: Predictors of new-onset decline in kidney function in a general middle-european population. Nephrol Dial Transplant 2008, 23:1265-1273.

28. Domrongkitchaiporn S, Sritara P, Kitiyakara C, Stitchantrakul W, Krittaphol V, Lolekha P, Cheepudomwit S, Yipintsoi T: Risk factors for development of decreased kidney function in a southeast Asian population: a 12-year cohort study. J Am Soc Nephrol 2005, 16:791-799.

29. Li YL, Wang L, Li J, Huang Y, Yuan WM: The correlation between uric acid and the incidence and prognosis of kidney diseases: a systematic review and meta-analysis of cohort studies. Zhonghua Nei Ke Za Zhi 2011, 50:555-561.

30. Chen LK, Lin MH, Lai HY, Hwang SJ, Chiou ST: Uric acid: a surrogate of insulin resistance in older women. Maturitas 2008, 59:55-61.

31. Xue C, Ye XD, Li W, Peng Q, Ding HY, Zhang YH, He DF, Bai X, Huang Y, Song YS, Pang L, Liao YH: Prevalence of chronic kidney disease in Jing adults in China: a village-based study. Clin Nephrol 2013, 79:50-56.

32. Feig DI, Soletsky B, Johnson RJ: Effect of allopurinol on blood pressure of adolescents with newly diagnosed essential hypertension: a randomized trial. JAMA 2008, 300:924-932.

33. Kanbay M, Ozkara A, Selcoki Y, Isik B, Turgut F, Bavbek N, Uz E, Akcay A, Yigitoglu R, Covic A: Effect of treatment of hyperuricemia with allopurinol on blood pressure, creatinine clearence, and proteinuria in patients with normal renal functions. Int Urol Nephrol 2007, 39:1227-1233.

34. Bose B, Badve SV, Hiremath SS, Boudville N, Brown FG, Cass A, de Zoysa JR, Fassett RG, Faull R, Harris DC, Hawley CM, Kanellis J, Palmer SC, Perkovic V, Pascoe EM, Rangan GK, Walker RJ, Walters G, Johnson DW: Effects of uric acid-lowering therapy on renal outcomes: a systematic review and meta-analysis. Nephrol Dial Transplant 2014, 29:406-413.

35. Thomas G, Sehgal AR, Kashyap SR, Srinivas TR, Kirwan JP, Navaneethan SD: Metabolic syndrome and kidney disease: a systematic review and meta-analysis. Clin J Am Soc Nephrol 2011, 6:2364-2373.

36. Palmer TM, Nordestgaard BG, Benn M, Tybjærg-Hansen A, Davey Smith G, Lawlor DA, Timpson NJ: Association of plasma uric acid with ischaemic heart disease and blood pressure: mendelian randomisation analysis of two large cohorts. BMJ 2013, 347:f4262.

37. Spencer HW, Yarger WE, Robinson RR: Alterations of renal function during dietary-induced hyperuricemia in the rat. Kidney Int 1976, 9:489-500.

38. Umekawa T, Chegini N, Khan SR: Increased expression of monocyte chemoattractant protein-1 (MCP-1) by renal epithelial cells in culture on 
exposure to calcium oxalate, phosphate and uric acid crystals. Nephrol Dial Transplant 2003, 18:664-669.

39. Mazzali M, Kanellis J, Han L, Feng L, Xia YY, Chen Q, Kang DH, Gordon KL, Watanabe S, Nakagawa T, Lan HY, Johnson RJ: Hyperuricemia induces a primary renal arteriolopathy in rats by a blood pressure-independent mechanism. Am J Physiol Renal Physiol 2002, 282:F991-F997.

40. Han HJ, Lim MJ, Lee YJ, Lee JH, Yang IS, Taub M: Uric acid inhibits renal proximal tubule cell proliferation via at least two signaling pathways involving PKC, MAPK, cPLA2, and NF-kappaB. Am J Physiol Renal Physiol 2007, 292:F373-F381

41. Kang DH, Nakagawa T, Feng L, Watanabe S, Han L, Mazzali M, Truong L, Harris $R$, Johnson RJ: A role for uric acid in the progression of renal disease. J Am Soc Nephrol 2002, 13:2888-2897.

42. Ryu ES, Kim MJ, Shin HS, Jang YH, Choi HS, Jo I, Johnson RJ, Kang DH: Uric acid-induced phenotypic transition of renal tubular cells as a novel mechanism of chronic kidney disease. Am J Physiol Renal Physiol 2013, 304:F471-F480.

doi:10.1186/1471-2369-15-122

Cite this article as: Li et al:: Is hyperuricemia an independent risk factor for new-onset chronic kidney disease?: a systematic review and meta-analysis based on observational cohort studies. BMC Nephrology 2014 15:122.

\section{Submit your next manuscript to BioMed Central and take full advantage of:}

- Convenient online submission

- Thorough peer review

- No space constraints or color figure charges

- Immediate publication on acceptance

- Inclusion in PubMed, CAS, Scopus and Google Scholar

- Research which is freely available for redistribution 